\section{TATRA \\ MOUNTaiNS \\ Mathematical Publications}

DOI: $10.2478 /$ tmmp-2013-0009

Tatra Mt. Math. Publ. 54 (2013), 101-118

\title{
THE STURM-LIOUVILLE PROBLEM WITH SINGULAR POTENTIAL AND THE EXTREMA OF THE FIRST EIGENVALUE
}

\author{
Elena S. Karulina-Anton A. Vladimirov
}

ABSTRACT. We get the infima and suprema of the first eigenvalue of the problem

$$
\begin{gathered}
-y^{\prime \prime}+q y=\lambda y, \\
\left\{\begin{array}{l}
y^{\prime}(0)-k_{0}^{2} y(0)=0, \\
y^{\prime}(1)+k_{1}^{2} y(1)=0,
\end{array}\right.
\end{gathered}
$$

where $q$ belongs to the set of constant-sign summable functions on $[0,1]$ such that

$$
\int_{0}^{1} q d x=1 \quad \text { or } \quad \int_{0}^{1} q d x=-1 .
$$

\section{Introduction}

1.1. Consider the Sturm-Liouville problem

$$
\begin{array}{r}
-y^{\prime \prime}+(q-\lambda) y=0, \\
\left\{\begin{array}{l}
y^{\prime}(0)-k_{0}^{2} y(0)=0, \\
y^{\prime}(1)+k_{1}^{2} y(1)=0,
\end{array}\right.
\end{array}
$$

where the real coefficients $k_{0} \geq 0$ and $k_{1} \geq k_{0}$ are fixed, the solution $y$ belongs to the space $W_{1}^{2}[0,1]$, the equality (11) is considered as holding almost everywhere at $[0,1]$, and the potential $q \in L_{1}[0,1]$ is a constant-sign function such that one

(C) 2013 Mathematical Institute, Slovak Academy of Sciences.

2010 Mathematics Subject Classification: 34L15.

Keywords: Sturm-Liouville problem, eigenvalue, Dirac delta function.

The first author is supported by the Russian Foundation for Basic Researches, grant

No 11-01-00989.

The second author is supported by the Russian Foundation for Basic Researches, grant No 10-01-00423. 
of the integral conditions holds:

$$
\int_{0}^{1} q d x=1 \quad \text { or } \quad \int_{0}^{1} q d x=-1 .
$$

The aim of this paper is to get the infima and suprema of the first eigenvalue of the problem (11)-(3).

1.2. The problem (1)-(3) is a partial case of the problem (11), (2) with $q \in A_{\gamma}$ or $-q \in A_{\gamma}$, where $\gamma \in \mathbb{R} \backslash\{0\}$ and

$$
A_{\gamma} \rightleftharpoons\left\{q \in L_{1}[0,1]: q(x) \geq 0 \text { a.e. and } \int_{0}^{1} q^{\gamma} d x=1\right\} \text {. }
$$

Denote by $\lambda_{1}(q)$ the minimal eigenvalue of the problem (11) or

$$
-y^{\prime \prime}-\lambda q y=0
$$

with some self-adjoint boundary conditions. Consider for each $\gamma \in \mathbb{R} \backslash\{0\}$ four values $m_{\gamma}^{ \pm} \rightleftharpoons \inf _{q \in A_{\gamma}} \lambda_{1}( \pm q)$ and $M_{\gamma}^{ \pm} \rightleftharpoons \sup _{q \in A_{\gamma}} \lambda_{1}( \pm q)$. The estimates of $m_{\gamma}^{+}$and $M_{\gamma}^{+}$for the equation (5) with the Dirichlet boundary conditions were obtained in [1]. The analogous results about the Dirichlet problem for the equation (1) were obtained in [2], 3]. In [4] the problem (5), (22) was studied.

The values $m_{\gamma}^{+}$and $M_{\gamma}^{+}$for the problem (1), (2) with $q \in A_{\gamma}$ were considered by one of the authors in $[5]$ for all $\gamma \neq 0$. The most detailed and precise results were obtained for the case $\gamma \neq 1$.

The case $\gamma=1$ is in some kind special. In [3] and [5], for (1) with various boundary conditions, the precise results for $M_{1}^{+}$were obtained by the method quite different from used for $\gamma \neq 1$. In [5] for $m_{1}^{+}$only inequality $m_{1}^{+} \geq 1 / 4$ was obtained. In [3] for $m_{1}^{-}$it was proved that this infimum is attained at the non-summable potential $q^{*}=-\boldsymbol{\delta}_{1 / 2}$.

In this paper we extend the class of considered potentials from $L_{1}[0,1]$ to the space $W_{2}^{-1}[0,1]$ (see [6] and 2.1 later). The space $W_{2}^{-1}[0,1]$, in particular, contains a Dirac delta function $\boldsymbol{\delta}_{\zeta}$ with support located at an arbitrary point $\zeta \in[0,1]$. This generalization of the problem lets us to get the precise description of $M_{1}^{-}$and $m_{1}^{ \pm}$and to prove that they are attained at the potentials from the extended class.

1.3. The main results of the paper are the following four theorems:

1.3.1. Theorem. By definition, put

$$
\alpha_{\mu} \rightleftharpoons \frac{1}{\sqrt{\mu}} \arctan \frac{k_{0}^{2}}{\sqrt{\mu}}, \quad \beta_{\mu} \rightleftharpoons \frac{1}{\sqrt{\mu}} \arctan \frac{k_{1}^{2}}{\sqrt{\mu}} .
$$


Then $M_{1}^{+}$is a unique solution to the equation

$$
1-\alpha_{\mu}-\beta_{\mu}=\mu^{-1}
$$

and is attained at the potential $q^{*} \in L_{1}[0,1]$ such that

$$
q^{*}(x)= \begin{cases}M_{1}^{+} & \text {for } x \in\left[\alpha_{M_{1}^{+}}, 1-\beta_{M_{1}^{+}}\right] \\ 0 & \text { otherwise. }\end{cases}
$$

1.3.2. TheOREM. If $k_{0}^{2}+k_{1}^{2} \leq 1$, then $M_{1}^{-}=k_{0}^{2}+k_{1}^{2}-1$ and is attained at the potential

$$
q^{*} \rightleftharpoons-k_{0}^{2} \boldsymbol{\delta}_{0}-k_{1}^{2} \boldsymbol{\delta}_{1}-\left(1-k_{0}^{2}-k_{1}^{2}\right) .
$$

If $k_{0}^{2}+k_{1}^{2} \geq 1$ and $k_{1}^{2}-k_{0}^{2} \leq 1$, then $M_{1}^{-}$is the minimal eigenvalue of the problem

$$
\begin{aligned}
-y^{\prime \prime} & =\lambda y \\
2 y^{\prime}(0)-\left(k_{0}^{2}+k_{1}^{2}-1\right) y(0) & =2 y^{\prime}(1)+\left(k_{0}^{2}+k_{1}^{2}-1\right) y(1)=0
\end{aligned}
$$

and is attained at the potential

$$
q^{*} \rightleftharpoons-\left(1+k_{0}^{2}-k_{1}^{2}\right) \boldsymbol{\delta}_{0} / 2-\left(1-k_{0}^{2}+k_{1}^{2}\right) \boldsymbol{\delta}_{1} / 2 .
$$

If $k_{1}^{2}-k_{0}^{2} \geq 1$, then $M_{1}^{-}$is the minimal eigenvalue of the problem (8) with

$$
y^{\prime}(0)-k_{0}^{2} y(0)=y^{\prime}(1)+\left(k_{1}^{2}-1\right) y(1)=0
$$

and is attained at the potential $q^{*} \rightleftharpoons-\boldsymbol{\delta}_{1}$.

1.3.3. TheOREM. $m_{1}^{+}$is the minimal eigenvalue of the problem (8) with

$$
y^{\prime}(0)-k_{0}^{2} y(0)=y^{\prime}(1)+\left(k_{1}^{2}+1\right) y(1)=0
$$

and is attained at the potential $q^{*} \rightleftharpoons \boldsymbol{\delta}_{1}$.

1.3.4. Theorem. If for some $\mu \geq-k_{0}^{4}$ and some $\zeta \in(0,1)$ the problem

$$
\begin{aligned}
-y^{\prime \prime} & =\mu y \quad \text { at }(0, \zeta) \cup(\zeta, 1), \\
y^{\prime}(0)-k_{0}^{2} y(0) & =2 y^{\prime}(\zeta-0)-y(\zeta) \\
& =2 y^{\prime}(\zeta+0)+y(\zeta)=y^{\prime}(1)+k_{1}^{2} y(1)=0
\end{aligned}
$$

has a continuous positive solution, then $m_{1}^{-}=\mu$ and $m_{1}^{-}$is attained at the potential $q^{*} \rightleftharpoons-\boldsymbol{\delta}_{\zeta}$. Otherwise $m_{1}^{-}$is the minimal eigenvalue of the problem (8) with

$$
y^{\prime}(0)-\left(k_{0}^{2}-1\right) y(0)=y^{\prime}(1)+k_{1}^{2} y(1)=0
$$

and is attained at the potential $q^{*} \rightleftharpoons-\boldsymbol{\delta}_{0}$.

Some additional remarks on solvability of the boundary problem (12), (13) will be given in the subsection 3.6 . 
1.4. Let us give some examples that illustrate the theorems from the previous subsection. In the case $k_{0}=k_{1}=0$ we get $m_{1}^{+}=\lambda_{1}\left(\boldsymbol{\delta}_{1}\right)=0.740174\left( \pm 10^{-6}\right)$. In the case $k_{0}^{2}=k_{1}^{2}>1 / 2$ we get $m_{1}^{-}=\lambda_{1}\left(-\boldsymbol{\delta}_{1 / 2}\right)$. In the case $k_{0}^{2}=k_{1}^{2}=1 / 2$ we have $m_{1}^{-}=\lambda_{1}\left(-\boldsymbol{\delta}_{\zeta}\right)=-1 / 4$ for any $\zeta \in[0,1]$. In the case $k_{0}^{2}=k_{1}^{2}<1 / 2$ we have $m_{1}^{-}=\lambda_{1}\left(-\boldsymbol{\delta}_{0}\right)$.

\section{The set $\Gamma_{1}$ and related topics}

2.1. We suppose that all considered functional spaces are real.

By $W_{2}^{-1}[0,1]$ denote the Hilbert space that is a completion of $L_{2}[0,1]$ in the norm

$$
\|y\|_{W_{2}^{-1}[0,1]} \rightleftharpoons \sup _{\|z\|_{W_{2}^{1}[0,1]}=1} \int_{0}^{1} y z d x .
$$

When $y \in W_{2}^{-1}[0,1]$, by $\int_{0}^{1} y z d x$ we sometimes denote the result

$$
\langle y, z\rangle \rightleftharpoons \lim _{n \rightarrow \infty} \int_{0}^{1} y_{n} z d x, \quad \text { where } \quad y=\lim _{n \rightarrow \infty} y_{n}, y_{n} \in L_{2}[0,1],
$$

of applying the linear functional $y$ to the function $z \in W_{2}^{1}[0,1]$.

For any fixed $q \in L_{1}[0,1]$ and $\lambda \in \mathbb{R}$ the map taking each $y \in W_{1}^{2}[0,1]$ satisfying (2) to

$$
-y^{\prime \prime}+(q-\lambda) y \in L_{1}[0,1]
$$

can be extended by continuity to the bounded operator $T_{q}(\lambda): W_{2}^{1}[0,1] \rightarrow$ $W_{2}^{-1}[0,1]$. Using integration by part, we get

$$
\begin{aligned}
\left(\forall y, z \in W_{2}^{1}[0,1]\right) \quad & \left\langle T_{q}(\lambda) y, z\right\rangle \\
& =\int_{0}^{1}\left[y^{\prime} z^{\prime}+(q-\lambda) y z\right] d x+k_{0}^{2} y(0) z(0)+k_{1}^{2} y(1) z(1) .
\end{aligned}
$$

Consider the linear operator penci $]^{1} T_{q}: \mathbb{R} \rightarrow \mathcal{B}\left(W_{2}^{1}[0,1], W_{2}^{-1}[0,1]\right)$ that takes any $\lambda \in \mathbb{R}$ to the operator $T_{q}(\lambda)$ described by (14). The spectral problem for $T_{q}$ may be considered as a reformulation (or as a generalization in case when $q \in W_{2}^{-1}[0,1]$ is not summable) of the boundary value problem (11), (2). We can do this due to the following two facts.

\footnotetext{
${ }^{1} \mathrm{~A}$ linear operator pencil $L$ is an operator-valued function such that $L(\lambda)=A+\lambda B$, where $\lambda \in \mathbb{R}, A$ and $B$ are some operators not depending on $\lambda$.
} 
2.1.1. For all $q \in L_{1}[0,1]$ and $\lambda \in \mathbb{R}$ the function $y \in W_{2}^{1}[0,1]$ belongs to the kernel of the operator $T_{q}(\lambda)$, if and only if $y \in W_{1}^{2}[0,1]$ and $y$ is a solution of the problem (11), (2).

P r o of. It directly follows from the definition of the operator $T_{q}(\lambda)$ that for any solution $y \in W_{1}^{2}[0,1]$ of the problem (1), (2) the equality $T_{q}(\lambda) y=0$ holds.

Let us prove the converse. Consider some $y \in \operatorname{ker} T_{q}(\lambda)$, and put

$$
w(x) \rightleftharpoons y^{\prime}(x)-\int_{0}^{x}(q-\lambda) y d t .
$$

For any $z \in \stackrel{\circ}{W_{2}^{1}}[0,1]$, using (14), we have

$$
0=\left\langle T_{q}(\lambda) y, z\right\rangle=\int_{0}^{1} w z^{\prime} d x .
$$

Since the set of the derivatives of all functions $z \in \stackrel{\circ}{W}_{2}^{1}[0,1]$ is an orthogonal complement in $L_{2}[0,1]$ of the set of all constants, from (16) it follows that the function $w \in L_{2}[0,1]$ is constant. Combining this with (15), we get that the function $y^{\prime}$ is absolutely continuous and its generalized derivative equals $(q-\lambda) y$. Now, using (14), we see that for any $z \in W_{2}^{1}[0,1]$ we get

$$
0=\left\langle T_{q}(\lambda) y, z\right\rangle=\left[-y^{\prime}(0)+k_{0}^{2} y(0)\right] z(0)+\left[y^{\prime}(1)+k_{1}^{2} y(1)\right] z(1),
$$

so $y$ satisfies the conditions (2).

2.1.2. For any $q \in W_{2}^{-1}[0,1]$ the spectrum of the linear operator pencil $T_{q}$ is purely discrete, simple and bounded from below.

Pr o of. Note that for any $y \in W_{2}^{1}[0,1]$ we have

$$
\left\|y^{2}\right\|_{W_{2}^{1}[0,1]} \leq \sup _{x \in[0,1]}|y(x)| \cdot \sqrt{\int_{0}^{1}\left[y^{2}+4\left(y^{\prime}\right)^{2}\right] d x} \leq 2\|y\|_{C[0,1]} \cdot\|y\|_{W_{2}^{1}[0,1]},
$$

then, by the embedding theorem, we get

$$
\left\|y^{2}\right\|_{W_{2}^{1}[0,1]} \leq C\|y\|_{W_{2}^{1}[0,1]}^{2},
$$

where $C$ is some constant.

Since $C[0,1]$ is densely embedded in $W_{2}^{-1}[0,1]$, for any $\varepsilon \in(0,1)$ there exists a function $\tilde{q} \in C[0,1]$ such that

$$
\|\tilde{q}-q\|_{W_{2}^{-1}[0,1]} \leq \varepsilon / C
$$


Using this and the inequality (17), for any $y \in W_{2}^{1}[0,1]$ we get

$$
\left|\int_{0}^{1}(\tilde{q}-q) y^{2} d x\right| \leq\|\tilde{q}-q\|_{W_{2}^{-1}[0,1]} \cdot\left\|y^{2}\right\|_{W_{2}^{1}[0,1]} \leq \varepsilon\|y\|_{W_{2}^{1}[0,1]}^{2} .
$$

Further, for any $\kappa>\|\tilde{q}\|_{C[0,1]}+1$ we have $\int_{0}^{1} \tilde{q} y^{2} d x \geq(1-\kappa) \int_{0}^{1} y^{2} d x$. Combining this with (14) and (18), we obtain

$$
I T_{q}(-\kappa) \geq 1-\varepsilon
$$

where by $I: W_{2}^{-1}[0,1] \rightarrow W_{2}^{1}[0,1]$ we denote an isometry that satisfies

$$
\left(\forall y \in W_{2}^{-1}[0,1]\right)\left(\forall z \in W_{2}^{1}[0,1]\right) \quad\langle I y, z\rangle_{W_{2}^{1}[0,1]}=\langle y, z\rangle .
$$

The existence and uniqueness of this isometry follows from the Riesz theorem about the representation of a functional in a Hilbert space [7, $§ 30, \S 99]$.

From the estimate (19) it follows [7, $\S 104]$ that the operator $S \rightleftharpoons I T_{q}(-\kappa)$ is boundedly invertible. Taking into account (14), we have $I T_{q}(\lambda) \equiv S-(\lambda+\kappa) J^{*} J$, where $J: W_{2}^{1}[0,1] \rightarrow L_{2}[0,1]$ is the embedding operator. So for any $\lambda \in \mathbb{R}$ the existence of a bounded inverse of the operator $T_{q}(\lambda)$ is equivalent to the existence of a bounded inverse of the operator $1-(\lambda+\kappa) S^{-1 / 2} J^{*} J S^{-1 / 2}$. Since $J$ is compact, it follows that the spectrum of $T_{q}$ is purely discrete, semi-simple and bounded from below.

The spectrum of the pencil $T_{q}$ is simple since (see [6], [8, Propositions 2, 10]) for any $\lambda \in \mathbb{R}$ the kernel of the operator $T_{q}(\lambda)$ is formed by the first components $Y_{1}$ of the solutions to the boundary value problem

$$
\begin{gathered}
\left(\begin{array}{l}
Y_{1} \\
Y_{2}
\end{array}\right)^{\prime}=\left(\begin{array}{cc}
u & 1 \\
-u^{2} & -u
\end{array}\right)\left(\begin{array}{l}
Y_{1} \\
Y_{2}
\end{array}\right), \\
Y_{2}(0)-k_{0}^{2} Y_{1}(0)=Y_{2}(1)+\left[k_{1}^{2}+\omega\right] Y_{1}(1)=0 .
\end{gathered}
$$

Here $u \in L_{2}[0,1]$ and $\omega \in \mathbb{R}$ are taken from the representation

$$
\left(\forall y \in W_{2}^{1}[0,1]\right) \quad \int_{0}^{1}(q-\lambda) y d x=-\int_{0}^{1} u y^{\prime} d x+\omega y(1)
$$

of the potential $q \in W_{2}^{-1}[0,1]$.

2.2. For the eigenvalues

$$
\lambda_{1}(q)<\lambda_{2}(q)<\cdots<\lambda_{n}(q)<\cdots
$$

of the pencil $T_{q}$ we have the following propositions. 
2.2.1. (See [8, Proposition 10].) For any $n \geq 1, q \in W_{2}^{-1}[0,1]$ and $\lambda \in \mathbb{R}$ the inequality $\lambda>\lambda_{n}(q)$ is equivalent to the existence of $n$-dimentional subspace $\mathfrak{N} \subset W_{2}^{1}[0,1]$ that satisfies

$$
(\forall y \in \mathfrak{N} \backslash\{0\}) \quad\left\langle T_{q}(\lambda) y, y\right\rangle<0 .
$$

2.2.2. For any $n \geq 1$ the function $\lambda_{n}: W_{2}^{-1}[0,1] \rightarrow \mathbb{R}$ is continuous.

Pr o of. Consider some $q \in W_{2}^{-1}[0,1]$ and $\varepsilon \in(0,1 / 2)$. For any $y \in W_{2}^{1}[0,1]$, $\lambda \in \mathbb{R}$ and $\tilde{q} \in W_{2}^{-1}[0,1]$ such that $\|\tilde{q}-q\|_{W_{2}^{-1}[0,1]}<\varepsilon / C$, where $C$ is the same as in (17), we get

$$
\begin{aligned}
\left\langle T_{\tilde{q}}(\lambda) y, y\right\rangle \geq & \left\langle T_{q}(\lambda) y, y\right\rangle-\varepsilon\|y\|_{W_{2}^{1}[0,1]}^{2} \\
\geq & \left\langle T_{q}(\lambda) y, y\right\rangle-\varepsilon\|y\|_{W_{2}^{1}[0,1]}^{2} \\
& \quad-\varepsilon \cdot\left\langle T_{2 q}\left(\lambda_{1}(2 q)\right) y, y\right\rangle-\varepsilon k_{0}^{2} y^{2}(0)-\varepsilon k_{1}^{2} y^{2}(1) \\
= & (1-2 \varepsilon) \cdot\left\langle T_{q}\left(\frac{\lambda+\varepsilon \cdot\left[1-\lambda_{1}(2 q)\right]}{1-2 \varepsilon}\right) y, y\right\rangle .
\end{aligned}
$$

Consequently, from the variational principle 2.2.1 it follows that any $\lambda>\lambda_{n}(\tilde{q})$ satisfies

$$
\frac{\lambda+\varepsilon \cdot\left[1-\lambda_{1}(2 q)\right]}{1-2 \varepsilon}>\lambda_{n}(q) .
$$

Since we can choose $\lambda$ arbitrarily close to $\lambda_{n}(\tilde{q})$, we have

$$
\lambda_{n}(\tilde{q}) \geq(1-2 \varepsilon) \lambda_{n}(q)-\varepsilon \cdot\left[1-\lambda_{1}(2 q)\right] .
$$

By the same method we get

$$
\lambda_{n}(\tilde{q}) \leq(1+2 \varepsilon) \lambda_{n}(q)+\varepsilon \cdot\left[1-\lambda_{1}(2 q)\right] .
$$

2.3. Let $\Gamma_{1}$ be the closure in $W_{2}^{-1}[0,1]$ of the set $A_{1}$ defined by (44). Put by definition

$$
\Lambda(X) \rightleftharpoons\left\{\lambda \in \mathbb{R}:(\exists q \in X) \quad \lambda=\lambda_{1}(q)\right\},
$$

where $X \subseteq W_{2}^{-1}[0,1]$ is some set of generalized functions. The set $\Lambda(X)$ is formed by all the possible values of $\lambda_{1}(q)$ for all $q \in X$. By $-X$ we, as usually, denote the set

$$
\left\{q \in W_{2}^{-1}[0,1]:(\exists r \in X) \quad q=-r\right\}
$$

2.3.1. Suppose $X$ is a dense subset of $\Gamma_{1}$, then the closures of $\Lambda( \pm X)$ and $\Lambda\left( \pm \Gamma_{1}\right)$ coincide. 
2.3.2. The extrema $m_{1}^{ \pm} \rightleftharpoons \inf \Lambda\left( \pm A_{1}\right)$ and $M_{1}^{ \pm} \rightleftharpoons \sup \Lambda\left( \pm A_{1}\right)$, defined in 1 .2. satisfy the equalities $m_{1}^{ \pm}=\inf \Lambda\left( \pm \Gamma_{1}\right)$ and $M_{1}^{ \pm}=\sup \Lambda\left( \pm \Gamma_{1}\right)$.

The proposition 2.3 .1 immediately follows from 2.2.2. The proposition 2.3 .2 immediately follows from 2.3.1.

2.3.3. The set $\Gamma_{1}$ consists of all non-negativ $\Theta^{2}$ distributions $q \in W_{2}^{-1}[0,1]$ such that $\int_{0}^{1} q d x=1$.

P r o of. Since for any $q \in \Gamma_{1}$ there exists a sequence of functions from $A_{1}$ such that its limit equals $q$, it follows that the generalized function $q$ is non-negative and satisfies $\int_{0}^{1} q d x=1$.

Let us prove the converse. Suppose $q \in W_{2}^{-1}[0,1]$ is a non-negative generalized function and satisfies $\int_{0}^{1} q d x=1$. Then (see [6], $[8, \S 2.3]$ ) there exists a function $u \in L_{2}[0,1]$ such that

$$
\left(\forall y \in W_{2}^{1}[0,1]\right) \quad \int_{0}^{1} q y d x=-\int_{0}^{1} u y^{\prime} d x+y(1) .
$$

Put by definition

$$
\Pi_{\gamma, \eta, \theta}(x) \rightleftharpoons \begin{cases}\frac{x-\gamma}{\eta-\gamma} & \text { for } x \in[\gamma, \eta], \\ \frac{\theta-x}{\theta-\eta} & \text { for } x \in[\eta, \theta], \\ 0 & \text { otherwise }\end{cases}
$$

for any reals $\gamma<\eta<\theta$. Suppose $0<a<b<c<d<1$. Substituting the functions $\Pi_{-1,0, a}+\Pi_{0, a, b}, \Pi_{a, b, c}+\Pi_{b, c, d}$ and $\Pi_{c, d, 1}+\Pi_{d, 1,2}$ for $y$ in (23), we get

$$
0 \leq \frac{1}{b-a} \int_{a}^{b} u d x \leq \frac{1}{d-c} \int_{c}^{d} u d x \leq 1 .
$$

From these inequalities it follows that the function $u \in L_{2}[0,1]$ is non-decreasing and satisfies vrai $\inf _{x \in[0,1]} u(x) \geq 0$ and vrai $\sup _{x \in[0,1]} u(x) \leq 1$.

Since there exists a sequence $\left\{u_{n}\right\}_{n=0}^{\infty}$ of non-decreasing piecewise linear functions such that $u_{n}(0)=0, u_{n}(1)=1$ and $u=\lim _{n \rightarrow \infty} u_{n}$, it follows that $q=\lim _{n \rightarrow \infty} u_{n}^{\prime}$, where $u_{n}^{\prime} \in A_{1}$.

2.4. Consider the function $F$ implicitely defined by the equation

$$
\lambda_{1}\left(F(\mu, \zeta) \boldsymbol{\delta}_{\zeta}\right)=\mu,
$$

where $\mu \in \mathbb{R}$ and $\zeta \in[0,1]$. The following three propositions give us some information about this function.

\footnotetext{
${ }^{2}$ The generalized function $q \in W_{2}^{-1}[0,1]$ is called non-negative if for any non-negative function $y \in W_{2}^{1}[0,1]$ the inequality $\langle q, y\rangle \geq 0$ holds.
} 
2.4.1. For any $\zeta \in[0,1]$ the function $F(\cdot, \zeta)$ is single-valued, strictly increasing, and its domain is the interval $\left(-\infty, f^{+}\right)$with some $f^{+}>0$.

Proof. For any $a \in \mathbb{R}$ there exists [8, Proposition 11] a positive eigenfunction $y \in \operatorname{ker} T_{a \boldsymbol{\delta}_{\zeta}}(\mu)$ corresponding to the eigenvalue $\mu \rightleftharpoons \lambda_{1}\left(a \boldsymbol{\delta}_{\zeta}\right)$, so for any $b<a$ we have

$$
\left\langle T_{b \boldsymbol{\delta}_{\zeta}}(\mu) y, y\right\rangle=\left\langle T_{a \boldsymbol{\delta}_{\zeta}}(\mu) y, y\right\rangle+(b-a) \cdot y^{2}(\zeta)<0 .
$$

Using 2.2.1, we now get $\lambda_{1}\left(b \boldsymbol{\delta}_{\zeta}\right)<\mu$. So the function $F(\cdot, \zeta)$ is the inverse of the strictly increasing and, according to 2.2.2, continuous map $a \mapsto \lambda_{1}\left(a \boldsymbol{\delta}_{\zeta}\right)$. Therefore, the function $F(\cdot, \zeta)$ is single-valued and strictly increasing.

Further, for any $a \in \mathbb{R}$ from the equality

$$
\left\langle T_{a \boldsymbol{\delta}_{\zeta}}\left(a+k_{0}^{2}+k_{1}^{2}\right) 1,1\right\rangle=a-\left(a+k_{0}^{2}+k_{1}^{2}\right)+k_{0}^{2}+k_{1}^{2}=0
$$

and the proposition 2.2.1 it follows that $\lambda_{1}\left(a \boldsymbol{\delta}_{\zeta}\right) \leq a+k_{0}^{2}+k_{1}^{2}$. Therefore, the domain of $F(\cdot, \zeta)$ is unbounded from below. Also for any $a>0$ we have $\lambda_{1}\left(a \boldsymbol{\delta}_{\zeta}\right)>0$, so the right bound of $\operatorname{dom} F(\cdot, \zeta)$ is positive.

2.4.2. The function $F$ is continuous.

Proof. Consider an arbitrary point $\left(\mu_{0}, \zeta_{0}\right) \in \operatorname{dom} F$ and suppose $a^{ \pm}$satisfy $a^{-}<F\left(\mu_{0}, \zeta_{0}\right)<a^{+}$. For any point $(\mu, \zeta) \in \mathbb{R} \times[0,1]$ sufficiently close to $\left(\mu_{0}, \zeta_{0}\right)$ from 2.4.1 and 2.2.2 we obtain the inequalities $\lambda_{1}\left(a^{-} \boldsymbol{\delta}_{\zeta}\right)<\mu<\lambda_{1}\left(a^{+} \boldsymbol{\delta}_{\zeta}\right)$. Hence there exists $a \in\left(a^{-}, a^{+}\right)$such that $\mu=\lambda_{1}\left(a \boldsymbol{\delta}_{\zeta}\right)$, so for the point $(\mu, \zeta)$ the equation (24) has a solution $F(\mu, \zeta)=a$.

2.4.3. A point $(\mu, \zeta) \in(0,+\infty) \times[0,1]$ belongs to domain of the function $F$ if and only if the following conditions hold:

$$
\sqrt{\mu} \cdot\left(\zeta-\alpha_{\mu}\right) \in(-\pi / 2, \pi / 2), \quad \sqrt{\mu} \cdot\left(1-\beta_{\mu}-\zeta\right) \in(-\pi / 2, \pi / 2),
$$

where $\alpha_{\mu}$ and $\beta_{\mu}$ are defined by (6). In this case the equality

$$
F(\mu, \zeta)=\sqrt{\mu} \cdot\left\{\tan \left[\sqrt{\mu} \cdot\left(\zeta-\alpha_{\mu}\right)\right]+\tan \left[\sqrt{\mu} \cdot\left(1-\beta_{\mu}-\zeta\right)\right]\right\}
$$

holds.

For any $\zeta \in[0,1]$ the equality

$$
F(0, \zeta)=-\frac{k_{0}^{2}}{1+k_{0}^{2} \zeta}-\frac{k_{1}^{2}}{1+k_{1}^{2}(1-\zeta)}
$$

holds.

For any $\mu<0$ and $\zeta \in[0,1]$ the equality

$$
F(\mu, \zeta)=-\sqrt{|\mu|} \cdot\left\{G\left(\sqrt{|\mu|}, k_{0}^{2}, \zeta\right)+G\left(\sqrt{|\mu|}, k_{1}^{2}, 1-\zeta\right)\right\}
$$


where

holds.

$$
G(\nu, \kappa, x) \rightleftharpoons \begin{cases}\tanh \left(\nu x+\ln \sqrt{\left.\frac{\nu+\kappa}{\nu-\kappa}\right)}\right. & \text { for } \nu>\kappa, \\ 1 & \text { for } \nu=\kappa, \\ \operatorname{coth}\left(\nu x+\ln \sqrt{\frac{\kappa+\nu}{\kappa-\nu}}\right) & \text { for } \nu<\kappa,\end{cases}
$$

Pr o of. Consider $\mu \in \mathbb{R}$ and $\zeta \in(0,1)$ such that $(\mu, \zeta) \in \operatorname{dom} F$. According to (20)-(22), the equality $T_{q}(\mu) y=0$, where $q \rightleftharpoons F(\mu, \zeta) \boldsymbol{\delta}_{\zeta}$, is equivalent to the boundary problem

$$
\begin{gathered}
-y^{\prime \prime}=\mu y \quad \text { at }(0, \zeta) \cup(\zeta, 1), \\
y^{\prime}(\zeta+0)-y^{\prime}(\zeta-0)=F(\mu, \zeta) y(\zeta), \\
y^{\prime}(0)-k_{0}^{2} y(0)=y^{\prime}(1)+k_{1}^{2} y(1)=0 .
\end{gathered}
$$

From [8, Proposition 11] and (24) it follows that any non-trivial solution to the problem (29)-(31) is constant-sign.

In the case $\mu>0$ any solution to the problem (29), (31) has the form

$$
y(x)= \begin{cases}A \cdot \cos \left[\sqrt{\mu} \cdot\left(1-\beta_{\mu}-\zeta\right)\right] \cdot \cos \left[\sqrt{\mu} \cdot\left(x-\alpha_{\mu}\right)\right] & \text { for } x<\zeta, \\ A \cdot \cos \left[\sqrt{\mu} \cdot\left(1-\beta_{\mu}-x\right)\right] \cdot \cos \left[\sqrt{\mu} \cdot\left(\zeta-\alpha_{\mu}\right)\right] & \text { for } x>\zeta,\end{cases}
$$

where $A$ is some constant. This function is constant-sign if and only if the conditions (25) hold. Using (30), we now get (26). The values $\zeta \in\{0,1\}$ are finally included in the consideration using the propositions 2.4 .2 and 2.2 .2 .

The cases $\mu=0$ and $\mu<0$ are considered on the base of (29)-(31) by analogous way using the solution

$$
y(x)= \begin{cases}A \cdot\left[1+k_{1}^{2}(1-\zeta)\right] \cdot\left[1+k_{0}^{2} x\right] & \text { for } x<\zeta, \\ A \cdot\left[1+k_{1}^{2}(1-x)\right] \cdot\left[1+k_{0}^{2} \zeta\right] & \text { for } x>\zeta\end{cases}
$$

in the case $\mu=0$, and the solution

$$
y(x)= \begin{cases}A \cdot g\left(\sqrt{|\mu|}, k_{1}^{2}, 1-\zeta\right) \cdot g\left(\sqrt{|\mu|}, k_{0}^{2}, x\right) & \text { for } x<\zeta, \\ A \cdot g\left(\sqrt{|\mu|}, k_{1}^{2}, 1-x\right) \cdot g\left(\sqrt{|\mu|}, k_{0}^{2}, \zeta\right) & \text { for } x>\zeta,\end{cases}
$$

where

in the case $\mu<0$.

$$
g(\nu, \kappa, x) \rightleftharpoons \begin{cases}\cosh \left(\nu x+\ln \sqrt{\frac{\nu+\kappa}{\nu-\kappa}}\right) & \text { for } \nu>\kappa, \\ e^{\nu x} & \text { for } \nu=\kappa, \\ \sinh \left(\nu x+\ln \sqrt{\frac{\kappa+\nu}{\kappa-\nu}}\right) & \text { for } \nu<\kappa,\end{cases}
$$




\section{Proofs of the main results}

3.1. In this section we prove Theorems 1.3.1 1.3.4. We use the notation

$$
\begin{aligned}
& \Omega^{+}(y) \rightleftharpoons\left\{x \in[0,1]: y(x)=\sup _{t \in[0,1]} y(t)\right\}, \\
& \Omega^{-}(y) \rightleftharpoons\left\{x \in[0,1]: y(x)=\inf _{t \in[0,1]} y(t)\right\},
\end{aligned}
$$

where $y \in W_{2}^{1}[0,1]$ is an arbitrary positive function. Also we take into account proposition 2.3.2.

3.2. Proof of Theorem 1.3.1, Consider some potential $q^{*} \in \Gamma_{1}$, and some positive eigenfunction $y \in \operatorname{ker} T_{q^{*}}\left(\lambda_{1}\left(q^{*}\right)\right)$. Suppose that the support of the generalized function $q^{*}$ is a subset of $\Omega^{+}(y)$. Then for any $q \in \Gamma_{1}$ we, using 2.3.3, have

$$
\begin{aligned}
0 & =\left\langle T_{q^{*}}\left(\lambda_{1}\left(q^{*}\right)\right) y, y\right\rangle \\
& =\int_{0}^{1}\left[\left(y^{\prime}\right)^{2}-\lambda_{1}\left(q^{*}\right) y^{2}\right] d x+\sup _{x \in[0,1]} y^{2}(x)+k_{0}^{2} y^{2}(0)+k_{1}^{2} y^{2}(1) \\
& \geq \int_{0}^{1}\left[\left(y^{\prime}\right)^{2}+\left(q-\lambda_{1}\left(q^{*}\right)\right) y^{2}\right] d x+k_{0}^{2} y^{2}(0)+k_{1}^{2} y^{2}(1)
\end{aligned}
$$

hence

$$
\left\langle T_{q}\left(\lambda_{1}\left(q^{*}\right)\right) y, y\right\rangle \leq 0
$$

It follows that $\lambda_{1}(q) \leq \lambda_{1}\left(q^{*}\right)$, therefore $\lambda_{1}\left(q^{*}\right)=M_{1}^{+}$. Thus we have proved that $M_{1}^{+}$is attained at any potential $q^{*}$ such that $\operatorname{supp} q^{*} \subseteq \Omega^{+}(y)$.

Suppose that $\Omega^{+}(y)=\left[\tau_{0}, \tau_{1}\right]$, where $\tau_{0} \neq \tau_{1}$. Also suppose that the potential $q^{*}$ is summable and has the form

$$
q^{*}(x)= \begin{cases}\mu & \text { for } x \in\left[\tau_{0}, \tau_{1}\right], \\ 0 & \text { otherwise, }\end{cases}
$$

where $\mu$ is some positive constant. Since $y^{\prime \prime}(x)=0$ for all $x \in\left(\tau_{0}, \tau_{1}\right)$, it follows that $\mu=\lambda_{1}\left(q^{*}\right)$. Therefore, the eigenfunction $y$ has the form

$$
y(x)= \begin{cases}A \cdot \cos \left[\sqrt{\mu} \cdot\left(x-\alpha_{\mu}\right)\right] & \text { for } x<\tau_{0}, \\ B & \text { for } x \in\left[\tau_{0}, \tau_{1}\right], \\ C \cdot \cos \left[\sqrt{\mu} \cdot\left(1-\beta_{\mu}-x\right)\right] & \text { for } x>\tau_{1},\end{cases}
$$

where $A, B$ and $C$ are some positive constants, and $\alpha_{\mu}, \beta_{\mu}$ are defined by (6). From the continuity of $y^{\prime}$ it follows that $\tau_{0}=\alpha_{\mu}$ and $\tau_{1}=1-\beta_{\mu}$, hence $A=B=C$. Finally, from the condition $\int_{0}^{1} q^{*} d x=1$ we have the equation (77). 
To conclude the proof, it remains to note that the equation (7) has a unique solution, because $\alpha_{\mu}$ and $\beta_{\mu}$, considered as functions of $\mu>0$, are non-negative, continuous, non-increasing and tend to zero as $\mu \rightarrow+\infty$.

3.3. Proof of Theorem 1.3.2. Consider some potential $q^{*} \in-\Gamma_{1}$, and some positive eigenfunction $y \in \operatorname{ker} T_{q^{*}}\left(\lambda_{1}\left(q^{*}\right)\right)$. Suppose that $\operatorname{supp} q^{*} \subseteq \Omega^{-}(y)$. Then for any $q \in-\Gamma_{1}$ we, using 2.3 .3 , have

$$
\begin{aligned}
0 & =\left\langle T_{q^{*}}\left(\lambda_{1}\left(q^{*}\right)\right) y, y\right\rangle \\
& =\int_{0}^{1}\left[\left(y^{\prime}\right)^{2}-\lambda_{1}\left(q^{*}\right) y^{2}\right] d x-\inf _{x \in[0,1]} y^{2}(x)+k_{0}^{2} y^{2}(0)+k_{1}^{2} y^{2}(1) \\
& \geq \int_{0}^{1}\left[\left(y^{\prime}\right)^{2}+\left(q-\lambda_{1}\left(q^{*}\right)\right) y^{2}\right] d x+k_{0}^{2} y^{2}(0)+k_{1}^{2} y^{2}(1),
\end{aligned}
$$

hence

$$
\left\langle T_{q}\left(\lambda_{1}\left(q^{*}\right)\right) y, y\right\rangle \leq 0
$$

It follows that $\lambda_{1}(q) \leq \lambda_{1}\left(q^{*}\right)$, therefore $\lambda_{1}\left(q^{*}\right)=M_{1}^{-}$. Thus we have proved that $M_{1}^{-}$is attained at any potential $q^{*}$ such that $\operatorname{supp} q^{*} \subseteq \Omega^{-}(y)$.

Suppose $k_{0}^{2}+k_{1}^{2} \leq 1$. Consider the generalized function

$$
q^{*} \rightleftharpoons-k_{0}^{2} \boldsymbol{\delta}_{0}-k_{1}^{2} \boldsymbol{\delta}_{1}-\left(1-k_{0}^{2}-k_{1}^{2}\right),
$$

which in this case belongs to $-\Gamma_{1}$. Using (14), we get that the first eigenfunction of the pencil $T_{q^{*}}$ is $y \equiv$ const, so $\operatorname{supp} q^{*} \subseteq \Omega^{-}(y)$. It follows that $M_{1}^{-}$is attained at the potential $q^{*}$ and is equal to the corresponding first eigenvalue

$$
\lambda_{1}\left(q^{*}\right)=k_{0}^{2}+k_{1}^{2}-1 .
$$

Suppose

$$
\begin{aligned}
& k_{0}^{2}+k_{1}^{2} \geq 1, \\
& k_{1}^{2}-k_{0}^{2} \leq 1 .
\end{aligned}
$$

Consider the generalized function $q^{*} \rightleftharpoons-\left(1+k_{0}^{2}-k_{1}^{2}\right) \boldsymbol{\delta}_{0} / 2-\left(1-k_{0}^{2}+k_{1}^{2}\right) \boldsymbol{\delta}_{1} / 2$, which, due to (36), belongs to $-\Gamma_{1}$. For such $q^{*}$ the equation $T_{q^{*}}(\lambda) y=0$ is equivalent to the problem (8) , (9). The first eigenvalue $\lambda_{1}\left(q^{*}\right)$, due to (35) and (9), is non-negative and the corresponding eigenfunction is

$$
y(x) \equiv \cos \left[\sqrt{\lambda_{1}\left(q^{*}\right)} \cdot(x-\zeta)\right],
$$

where $\zeta=1 / 2$. Hence $\operatorname{supp} q^{*} \subseteq \Omega^{-}(y)$. It follows that $M_{1}^{-}$is attained at the potential $q^{*}$ and is equal to the corresponding first eigenvalue $\lambda_{1}\left(q^{*}\right)$.

Suppose $k_{1}^{2}-k_{0}^{2} \geq 1$. Consider the generalized function $q^{*} \rightleftharpoons-\boldsymbol{\delta}_{1} \in-\Gamma_{1}$. For such $q^{*}$ the equation $T_{q^{*}}(\lambda) y=0$ is equivalent to the problem (8), (10). 
The corresponding first eigenfunction is defined by (37), where $\zeta \in[0,1 / 2]$, since $k_{1}^{2}-1 \geq k_{0}^{2}$. Hence $\operatorname{supp} q^{*} \subseteq \Omega^{-}(y)$. It follows that $M_{1}^{-}$is attained at the potential $q^{*}$ and is equal to the corresponding first eigenvalue $\lambda_{1}\left(q^{*}\right)$.

3.4. Proof of Theorem 1.3.3. Consider some potential $q \in \Gamma_{1}$, and some positive eigenfunction $y \in \operatorname{ker} T_{q}\left(\lambda_{1}(q)\right)$. Then for any $\lambda>\lambda_{1}(q)$, according to 2.3.3, we have

$$
\begin{aligned}
0 & >\int_{0}^{1}\left[\left(y^{\prime}\right)^{2}+(q-\lambda) y^{2}\right] d x+k_{0}^{2} y^{2}(0)+k_{1}^{2} y^{2}(1) \\
& \geq \int_{0}^{1}\left[\left(y^{\prime}\right)^{2}-\lambda y^{2}\right] d x+\inf _{x \in[0,1]} y^{2}(x)+k_{0}^{2} y^{2}(0)+k_{1}^{2} y^{2}(1) .
\end{aligned}
$$

It follows that there exists $\zeta \in[0,1]$ such that

$$
\int_{0}^{1}\left[\left(y^{\prime}\right)^{2}+\left(\boldsymbol{\delta}_{\zeta}-\lambda\right) y^{2}\right] d x+k_{0}^{2} y^{2}(0)+k_{1}^{2} y^{2}(1)<0 .
$$

So for any $\lambda>m_{1}^{+}$there exists $\zeta \in[0,1]$ such that $\lambda_{1}\left(\boldsymbol{\delta}_{\zeta}\right)<\lambda$. Hence, using 2.3.3, we get $m_{1}^{+}=\inf _{x \in[0,1]} \lambda_{1}\left(\boldsymbol{\delta}_{x}\right)$. This equality is equivalent, according to 2.4.1, to the following fact: $F\left(m_{1}^{+}, x\right)$ is defined for all $x \in[0,1]$ and satisfies $\sup _{x \in[0,1]} F\left(m_{1}^{+}, x\right)=1$.

Since $m_{1}^{+}>0$, from 2.4 .3 it follows that if $\mu=m_{1}^{+}$, then for any $\zeta \in[0,1]$ the conditions (25) hold. According to (26), (25) and

$$
\frac{\partial F(\mu, \zeta)}{\partial \zeta} \equiv \mu \cdot \frac{\cos ^{2}\left[\sqrt{\mu} \cdot\left(1-\beta_{\mu}-\zeta\right)\right]-\cos ^{2}\left[\sqrt{\mu} \cdot\left(\zeta-\alpha_{\mu}\right)\right]}{\cos ^{2}\left[\sqrt{\mu} \cdot\left(\zeta-\alpha_{\mu}\right)\right] \cdot \cos ^{2}\left[\sqrt{\mu} \cdot\left(1-\beta_{\mu}-\zeta\right)\right]},
$$

it follows that the function $F(\mu, \cdot)$ can have at some point $\zeta \in(0,1)$ a local extremum satisfying $F(\mu, \zeta)>0$ only if $\zeta=\left(1-\beta_{\mu}+\alpha_{\mu}\right) / 2, \zeta>\alpha_{\mu}$ and $\zeta<1-\beta_{\mu}$. But this conditions imply, according to (38), that such $\zeta$ must be a point of strict local minimum of the function $F(\mu, \cdot)$. Therefore, $F(\mu, \cdot)$ cannot have a supremum in $(0,1)$, so we get $m_{1}^{+}=\inf \left\{\lambda_{1}\left(\boldsymbol{\delta}_{0}\right), \lambda_{1}\left(\boldsymbol{\delta}_{1}\right)\right\}$. Note that for the potential $q^{*} \rightleftharpoons \boldsymbol{\delta}_{i}$, where $i \in\{0,1\}$, the equation $T_{q^{*}}(\lambda) y=0$ is equivalent to the problem

$$
\begin{aligned}
-y^{\prime \prime} & =\lambda y \\
y^{\prime}(0)-\left[k_{0}^{2}+(1-i)\right] y(0) & =y^{\prime}(1)+\left[k_{1}^{2}+i\right] y(1)=0 .
\end{aligned}
$$

Therefore, we have

so $m_{1}^{+}=\lambda_{1}\left(\boldsymbol{\delta}_{1}\right)$.

$$
\frac{\lambda_{1}\left(\boldsymbol{\delta}_{i}\right)-k_{0}^{2} k_{1}^{2}-k_{1-i}^{2}}{k_{0}^{2}+k_{1}^{2}+1}=\sqrt{\lambda_{1}\left(\boldsymbol{\delta}_{i}\right)} \cot \sqrt{\lambda_{1}\left(\boldsymbol{\delta}_{i}\right)},
$$


3.5. Proof of Theorem 1.3.4. Consider some potential $q \in-\Gamma_{1}$, and some positive eigenfunction $y \in \operatorname{ker} T_{q}\left(\lambda_{1}(q)\right)$. Then for any $\lambda>\lambda_{1}(q)$, according to 2.3.3, we have

$$
\begin{aligned}
0 & >\int_{0}^{1}\left[\left(y^{\prime}\right)^{2}+(q-\lambda) y^{2}\right] d x+k_{0}^{2} y^{2}(0)+k_{1}^{2} y^{2}(1) \\
& \geq \int_{0}^{1}\left[\left(y^{\prime}\right)^{2}-\lambda y^{2}\right] d x-\sup _{x \in[0,1]} y^{2}(x)+k_{0}^{2} y^{2}(0)+k_{1}^{2} y^{2}(1) .
\end{aligned}
$$

It follows that there exists $\zeta \in[0,1]$ such that

$$
\int_{0}^{1}\left[\left(y^{\prime}\right)^{2}+\left(-\boldsymbol{\delta}_{\zeta}-\lambda\right) y^{2}\right] d x+k_{0}^{2} y^{2}(0)+k_{1}^{2} y^{2}(1)<0 .
$$

So for any $\lambda>m_{1}^{-}$there exists $\zeta \in[0,1]$ such that $\lambda_{1}\left(-\boldsymbol{\delta}_{\zeta}\right)<\lambda$. Hence, using 2.3.3. we get $m_{1}^{-}=\inf _{x \in[0,1]} \lambda_{1}\left(-\boldsymbol{\delta}_{x}\right)$. This equality is equivalent, according to 2.4.1, to the following fact: $F\left(m_{1}^{-}, x\right)$ is defined for all $x \in[0,1]$ and satisfies $\sup _{x \in[0,1]} F\left(m_{1}^{-}, x\right)=-1$.

For any fixed value $\mu \in \mathbb{R}$ we consider the conditions

$$
\begin{aligned}
F(\mu, \zeta) & <0, \\
\partial F(\mu, \zeta) / \partial \zeta & =0 .
\end{aligned}
$$

It is clear that some point $\zeta \in(0,1)$ can satisfy the equalities $F(\mu, \zeta)=$ $\sup _{x \in[0,1]} F(\mu, x)=-1$ only if (39) and (40) hold.

Suppose $\mu>0$. Then, according to (38), (26), (32) and (30), for any point $\zeta \in(0,1)$ satisfying (39) the condition (40) holds if and only if the problem

$$
\begin{aligned}
-y^{\prime \prime} & =\mu y \quad \text { at }(0, \zeta) \cup(\zeta, 1), \\
y^{\prime}(0)-k_{0}^{2} y(0) & =2 y^{\prime}(\zeta-0)+F(\mu, \zeta) y(\zeta) \\
& =2 y^{\prime}(\zeta+0)-F(\mu, \zeta) y(\zeta)=y^{\prime}(1)+k_{1}^{2} y(1)=0
\end{aligned}
$$

has a continuous positive solution. Besides, for any point $\zeta \in(0,1)$ satisfying (39) and (40) we have

$$
\alpha_{\mu}>\zeta>1-\beta_{\mu} .
$$

Therefore, according to (38) and (25), this stationary point $\zeta$ is a strict maximum of $F(\mu, \cdot)$. Since for any $x \in[0,1]$, using (43), we get

$$
-\pi / 2<-\sqrt{\mu} \alpha_{\mu} \leq \sqrt{\mu} \cdot\left(x-\alpha_{\mu}\right)<\sqrt{\mu} \cdot\left(x-1+\beta_{\mu}\right) \leq \sqrt{\mu} \beta_{\mu}<\pi / 2,
$$

it follows from the proposition 2.4 .3 that the function $F(\mu, \cdot)$ is defined everywhere on $[0,1]$. 


\section{THE EXTREMA OF STURM-LIOUVILLE EIGENVALUE}

Suppose $\mu=0$. Let us use the same method as in the previous case, changing (26) to (27), and (32) to (33). Then we get that for any point $\zeta \in(0,1)$ satisfying (39) the condition (40) holds if and only if the problem (41), (42) has a continuous positive solution. Using (27) we also get that the second derivative of $F(0, \cdot)$ is negative. Hence any stationary point $\zeta \in(0,1)$ is a strict maximum of $F(0, \cdot)$.

Suppose $\mu \in\left(-k_{0}^{4}, 0\right)$. Then, using (28), we get

$$
\frac{\partial F(\mu, \zeta)}{\partial \zeta} \equiv-\mu\left\{\sinh ^{-2}\left(\sqrt{|\mu|} \zeta+\alpha_{\mu}\right)-\sinh ^{-2}\left(\sqrt{|\mu|}(1-\zeta)+\beta_{\mu}\right)\right\}
$$

where

$$
\alpha_{\mu} \rightleftharpoons \frac{1}{2} \ln \frac{k_{0}^{2}+\sqrt{|\mu|}}{k_{0}^{2}-\sqrt{|\mu|}}, \quad \beta_{\mu} \rightleftharpoons \frac{1}{2} \ln \frac{k_{1}^{2}+\sqrt{|\mu|}}{k_{1}^{2}-\sqrt{|\mu|}} .
$$

Therefore, according to (34), for any point $\zeta \in(0,1)$ satisfying (39) the condition (40) holds if and only if the problem (41), (42) has a continuous positive solution. Since $\partial^{2} F(\mu, \zeta) / \partial \zeta^{2}<0$, it follows that any stationary point $\zeta \in(0,1)$ is a strict maximum of $F(\mu, \cdot)$.

Suppose $0>\mu=-k_{0}^{4}=-k_{1}^{4}$. Then the function $F(\mu, \cdot)$ is a negative constant, and for any point $\zeta \in(0,1)$ problem (41), (42) has a continuous positive solution.

Suppose $\mu \in\left[-k_{1}^{4},-k_{0}^{4}\right]$, also $\mu<0$ and $k_{1}>k_{0}$. Then from (28) and (34) it follows that $\partial F(\mu, \zeta) / \partial \zeta<0$, and the problem (41), (42) has no positive solutions for any $\zeta \in(0,1)$.

Suppose $\mu<-k_{1}^{4}$. Then, using (28), we get

$$
\frac{\partial F(\mu, \zeta)}{\partial \zeta} \equiv \mu\left\{\cosh ^{-2}\left(\sqrt{|\mu|} \zeta+\alpha_{\mu}\right)-\cosh ^{-2}\left(\sqrt{|\mu|}(1-\zeta)+\beta_{\mu}\right)\right\}
$$

where

$$
\alpha_{\mu} \rightleftharpoons \frac{1}{2} \ln \frac{\sqrt{|\mu|}+k_{0}^{2}}{\sqrt{|\mu|}-k_{0}^{2}}, \quad \beta_{\mu} \rightleftharpoons \frac{1}{2} \ln \frac{\sqrt{|\mu|}+k_{1}^{2}}{\sqrt{|\mu|}-k_{1}^{2}} .
$$

Therefore, according to (34), for any point $\zeta \in(0,1)$ satisfying (39) the condition (40) holds if and only if problem (41), (42) has a continuous positive solution. Since $\partial^{2} F(\mu, \zeta) / \partial \zeta^{2}>0$, it follows that any stationary point $\zeta \in(0,1)$ is a strict minimum of $F(\mu, \cdot)$.

From the proposition 2.4.1 we also get that for any $\mu \leq 0$ the function $F(\mu, \cdot)$ is defined everywhere on $[0,1]$.

Combining all this, we obtain the following: the existence of a continuous positive solution to the problem (12), (13) for some $\mu \geq-k_{0}^{4}$ and $\zeta \in(0,1)$ implies that $F(\mu, \zeta)=-1$, the function $F(\mu, \cdot)$ is defined everywhere on $[0,1]$, and $\sup _{x \in[0,1]} F(\mu, x) \leq-1$. Therefore, $m_{1}^{-}=\lambda_{1}\left(-\boldsymbol{\delta}_{\zeta}\right)$. In converse, if for any $\mu \geq-k_{0}^{4}$ and $\zeta \in(0,1)$ the positive solution of (12), (13) does not exist, we get $m_{1}^{-}=\inf \left\{\lambda_{1}\left(-\boldsymbol{\delta}_{0}\right), \lambda_{1}\left(-\boldsymbol{\delta}_{1}\right)\right\}$. From the equation

$$
\lambda_{1}\left(-\boldsymbol{\delta}_{i}\right)-k_{0}^{2} k_{1}^{2}+k_{1-i}^{2}=\left(k_{0}^{2}+k_{1}^{2}-1\right) \cdot \psi\left(\lambda_{1}\left(-\boldsymbol{\delta}_{i}\right)\right),
$$


where $i \in\{0,1\}$ and

$$
\psi(x) \rightleftharpoons \begin{cases}\sqrt{x} \cot \sqrt{x} & \text { for } x>0, \\ 1 & \text { for } x=0, \\ \sqrt{|x|} \operatorname{coth} \sqrt{|x|} & \text { for } x<0,\end{cases}
$$

we obtain that $\inf \left\{\lambda_{1}\left(-\boldsymbol{\delta}_{0}\right), \lambda_{1}\left(-\boldsymbol{\delta}_{1}\right)\right\}=\lambda_{1}\left(-\boldsymbol{\delta}_{0}\right)$.

3.6. Now we get some conditions for the existence of a continuous positive solution to the problem (12), (13) considered in Theorem 1.3.4.

Suppose $\mu_{0}(\zeta)$, where $\zeta \in(0,1]$, is the minimal eigenvalue of the problem

$$
\begin{aligned}
-y^{\prime \prime} & =\lambda y, \\
y^{\prime}(0)-k_{0}^{2} y(0) & =2 y^{\prime}(\zeta)-y(\zeta)=0,
\end{aligned}
$$

and suppose $\mu_{1}(\zeta)$, where $\zeta \in[0,1)$, is the minimal eigenvalue of the problem (44) and

$$
2 y^{\prime}(\zeta)+y(\zeta)=y^{\prime}(1)+k_{1}^{2} y(1)=0 .
$$

It is clear that for some $\mu \in \mathbb{R}$ and $\zeta \in(0,1)$ a continuous positive solution to (12), (13) exists if and only if the equalities $\mu_{0}(\zeta)=\mu_{1}(\zeta)=\mu$ hold.

3.6.1. If $k_{0}^{2}=1 / 2$, then $\mu_{0}(\zeta) \equiv-1 / 4$.

If $k_{0}^{2}>1 / 2$, then the function $\mu_{0}$ strictly decreases and satisfies

$$
\lim _{\zeta \rightarrow 0} \mu_{0}(\zeta)=+\infty \quad \text { and } \quad \mu_{0}(1)>-1 / 4
$$

If $k_{0}^{2}<1 / 2$, then for any $\zeta \in(0,1]$ the inequality $\mu_{0}(\zeta)<-1 / 4$ holds.

Pr o of. Suppose $k_{0}^{2}=1 / 2$. Then for any $\zeta \in(0,1]$ the problem (44), (45) has the positive eigenfunction $y(x) \equiv e^{x / 2}$ corresponding to the eigenvalue $-1 / 4$.

Suppose $k_{0}^{2}>1 / 2$. Since the eigenvalues of the problem (44), (45) increase by $k_{0}^{2}$, it follows that $\mu_{0}(\zeta)>-1 / 4$. Then let $y_{0} \in W_{2}^{1}[0, \zeta]$ be an eigenfunction of the problem (44), (45) corresponding to the eigenvalue $\mu_{0}(\zeta)$. Continuing the function $y_{0}$ for any $\theta \in(\zeta, 1]$ to the interval $(\zeta, \theta]$ in the form $y(x) \rightleftharpoons$ $y_{0}(\zeta) e^{(x-\zeta) / 2}$, for the obtained function $y \in W_{2}^{1}[0, \theta]$ we get

$$
\begin{aligned}
\int_{0}^{\theta}\left[\left(y^{\prime}\right)^{2}-\mu_{0}(\zeta) y^{2}\right] d x+k_{0}^{2} y^{2}(0) & -\frac{y^{2}(\theta)}{2} \\
& =\left[-1 / 4-\mu_{0}(\zeta)\right] \cdot\left[e^{\theta-\zeta}-1\right] \cdot y^{2}(\zeta)<0
\end{aligned}
$$

hence $\mu_{0}(\theta)<\mu_{0}(\zeta)$. 
Finally, for $\zeta \rightarrow 0$ we have uniform by $y \in W_{2}^{1}[0, \zeta]$ asymptotic estimate

$$
\begin{aligned}
& \int_{0}^{\zeta}\left(y^{\prime}\right)^{2} d x+k_{0}^{2} y^{2}(0)-\frac{y^{2}(\zeta)}{2} \\
& =\left[\int_{0}^{\zeta} \frac{\left(y^{\prime}\right)^{2}}{2} d x+\left(k_{0}^{2}-1 / 2\right) y^{2}(0)\right]+\left[\int_{0}^{\zeta} \frac{\left(y^{\prime}\right)^{2}}{2} d x+\frac{y^{2}(0)-y^{2}(\zeta)}{2}\right] \\
& \geq \frac{k_{0}^{2}-1 / 2+o(1)}{\zeta} \int_{0}^{\zeta} y^{2} d x-\frac{1}{2} \int_{0}^{\zeta} y^{2} d x
\end{aligned}
$$

therefore, $\mu_{0}(\zeta) \geq\left[k_{0}^{2}-1 / 2+o(1)\right] \cdot \zeta^{-1}$.

The inequality $\mu_{0}(\zeta)<-1 / 4$ for the case $k_{0}^{2}<1 / 2$ is proved likewise the inequality $\mu_{0}(\zeta)>-1 / 4$ for the case $k_{0}^{2}>1 / 2$.

3.6.2. If $k_{1}^{2}=1 / 2$, then $\mu_{1}(\zeta) \equiv-1 / 4$.

If $k_{1}^{2}>1 / 2$, then the function $\mu_{1}$ strictly increases and satisfies

$$
\lim _{\zeta \rightarrow 1} \mu_{1}(\zeta)=+\infty \text { and } \mu_{1}(0)>-1 / 4 .
$$

If $k_{1}^{2}<1 / 2$, then for any $\zeta \in[0,1)$ the inequality $\mu_{1}(\zeta)<-1 / 4$ holds.

The proposition 3.6 .2 is proved likewise 3.6.1.

Combining 3.6.1 and 3.6.2, we get the last proposition:

3.6.3. The problem (12), (13) has a continuous positive solution for some $\mu \geq-k_{0}^{4}$ and $\zeta \in(0,1)$ if and only if this condition holds:

$$
k_{0}^{2}>1 / 2 \quad \text { or } \quad k_{0}^{2}=k_{1}^{2}=1 / 2 .
$$

\section{REFERENCES}

[1] EGOROV, YU. V.-KONDRATIEV, V. A.: On Spectral Theory of Elliptic Operators, in: Oper. Theory Adv. Appl., Vol. 89, Birkhäuser, 1996.

[2] VINOKUROV, V. A.-SADOVNICHII, V. A.: On the range of variation of an eigenvalue when potential is varied, Dokl. Math. 68 (2003), 247-252.

[3] EZHAK, S. S.: On the estimates for the minimum eigenvalue of the Sturm-Liouville problem with integral condition, J. Math. Sci. 145 (2007), 5205-5218.

[4] MURYSHKINA, O. V.: Estimates for the minimal eigenvalue of the Sturm-Liouville problem with nonsymmetric boundary conditions, Differ. Equ. 37 (2001), 899-900.

[5] KARULINA, E.: Some estimates for the minimal eigenvalue of the Sturm-Liouville problem with third-type boundary conditions, Math. Bohemica 136 (2011), 377-384. 


\section{ELENA S. KARULINA-ANTON A. VLADIMIROV}

[6] SAVCHUK, A. M.-SHKALIKOV, A. A.: Sturm-Liouville operators with distribution potentials, Trans. Moscow Math. Soc. 64 (2003), 143-192.

[7] RIESZ, F.-NAGY, B. SZ.: Leçons d'analyse fonctionelle. Budapest, Akadémiai Kiadó, 1968.

[8] VLADIMIROV, A. A.: On the oscillation theory of the Sturm-Liouville problem with singular coefficients, Comput. Math. Math. Phys. 49 (2009), 1535-1546.

Received July 2, 2012

Elena S. Karulina

Moscow State University of Economics

Statistics and Informatics

Nezhinskaja 7

Moscow 119501

RUSSIA

E-mail: karulinaes@yandex.ru

Anton A. Vladimirov

Dorodnitsyn Computing Center of RAS

Vavilova 40

Moscow 119991

RUSSIA

E-mail: vladimi@mech.math.msu.su 